\title{
A remark on the Tribonacci sequences
}

\author{
Lilija Atanassova \\ Institute of Information and Communication Technologies \\ Bulgarian Academy of Sciences \\ Acad. G. Bonchev Str., Bl. 2, Sofia-1113, Bulgaria \\ e-mail: 1.c.atanassova@gmail.com
}

Received: 30 April 2019

Accepted: 18 August 2019

\begin{abstract}
One of the first extensions of the Fibonacci sequence are the Tribonacci sequences. In the paper, some of their properties are discussed.
\end{abstract}

Keywords: Tribonacci sequence, Natural number.

2010 Mathematics Subject Classification: 11B39.

\section{Introduction and main result}

The well-known Fibonacci sequence is an object of a lot extensions. One of the first of them is the Tribonacci sequence, see [6], introduced in 1963 by Mark Feinberg, a fourteen-year-old student in the ninth grade of the Susquehanna Township Junior High School (USA). He introduced the Tribonacci sequence in three different forms. The first of them is:

$1,1,1,3,5,9,17,31,57,105,193,355,653,1201,2209,4063,7473,13745,25281,46499$, $85525,157305,289329,532159,978793,1800281,3311233,6090307,11201821, \ldots$

The second form, which is very often used (see, e.g., [9]), is $0,0,1,1,2,4,7,13,24,44,81,149,274,504,927,1705,3136,5768,10609,19513,35890$, 66012, 121415, 223317, 410744, 755476, 1389537, 2555757, 4700770, 8646064, 15902591, 29249425, 53798080, 98950096, 181997601, 334745777, 615693474, 1132436852,2082876103, $3831006429, \ldots$

In practice, the third form coincides up to the enumeration with the second one:

$0,1,1,2,4,7,13,24,44,81,149,274,504,927,1705,3136,5768,10609,19513,35890,66012$, 121415, 223317, 410744, 755476, 1389537, 2555757, 4700770, 8646064, 15902591, 29249425, 
53798080, 98950096, 181997601, 334745777, 615693474, 1132436852, 2082876103,

$3831006429, \ldots$

The formula for the $n$-th number is given by A. Shannon in [8].

Let the elements of each of the three Tribonacci sequence forms be denoted as $\left\{T_{i}\right\}_{i \geq 0}$.

Theorem 1. For each natural number $n \geq 0$,

$$
T_{n}+T_{n+13}+T_{n+26} \equiv 0(\bmod 3) .
$$

Proof. Let $n=0,1,2$. Then, for the first Tribonacci sequence form we obtain

$$
\begin{gathered}
T_{0}+T_{13}+T_{26}=1+1201+3311233=3312435 \equiv 0(\bmod 3), \\
T_{1}+T_{14}+T_{27}=1+2209+6090307=6092517 \equiv 0(\bmod 3), \\
T_{2}+T_{15}+T_{28}=1+4063+11201821=11205885 \equiv 0(\bmod 3) .
\end{gathered}
$$

For the second Tribonacci sequence form we obtain

$$
\begin{gathered}
T_{0}+T_{13}+T_{26}=0+504+1389537=1390041 \equiv 0(\bmod 3), \\
T_{1}+T_{14}+T_{27}=0+927+2555757=2556684 \equiv 0(\bmod 3), \\
T_{2}+T_{15}+T_{28}=1+1705+4700770=11205885 \equiv 0(\bmod 3) .
\end{gathered}
$$

For the third Tribonacci sequence form we obtain

$$
\begin{gathered}
T_{0}+T_{13}+T_{26}=0+927+2555757=2556684 \equiv 0(\bmod 3), \\
T_{1}+T_{14}+T_{27}=1+1705+4700770=11205885 \equiv 0(\bmod 3), \\
T_{2}+T_{15}+T_{28}=1+3136+8646064=8649201 \equiv 0(\bmod 3) .
\end{gathered}
$$

Let us assume that the assertion is valid for each of the three Tribonacci sequence forms and for the natural numbers less than or equal to $n+2$, i.e.,

$$
\begin{gathered}
T_{n}+T_{n+13}+T_{n+26} \equiv 0(\bmod 3), \\
T_{n+1}+T_{n+14}+T_{n+27} \equiv 0(\bmod 3), \\
T_{n+2}+T_{n+15}+T_{n+28} \equiv 0(\bmod 3) .
\end{gathered}
$$

Then

$$
\begin{gathered}
T_{n+3}+T_{n+16}+T_{n+29} \\
=\left(T_{n}+T_{n+1}+T_{n+2}\right)+\left(T_{n+13}+T_{n+14}+T_{n+15}\right)+\left(T_{n+26}+T_{n+27}+T_{n+28}\right) \\
=\left(T_{n}+T_{n+13}+T_{n+26}\right)+\left(T_{n+1}+T_{n+14}+T_{n+27}\right)+\left(T_{n+2}+T_{n+15}+T_{n+28}\right) \\
\equiv 0(\bmod 3) .
\end{gathered}
$$


It is suitable to re-write the above sequences as follows

$\begin{array}{lllllllllllll}1 & 1 & 1 & 3 & 5 & 9 & 17 & 31 & 57 & 105 & 193 & 355 & 653\end{array}$ 1201220940637473137452528146499855251573052893295321599787931800281

$\begin{array}{lllllllllllll}0 & 0 & 1 & 1 & 2 & 4 & 7 & 13 & 24 & 44 & 81 & 149 & 274\end{array}$ 50492717053136576810609195133589066012121415223317410744755476

$\begin{array}{lllllllllllll}0 & 1 & 1 & 2 & 4 & 7 & 13 & 24 & 44 & 81 & 149 & 274 & 504\end{array}$ 927170531365768106091951335890660121214152233174107447554761389537

respectively. Now, we can prove by the above manner that for each natural number $n \geq 0$ for the first sequence are valid:

$$
\begin{aligned}
& T_{13 n+3} \equiv 0(\bmod 3), \\
& T_{13 n+5} \equiv 0(\bmod 9), \\
& T_{13 n+8} \equiv 0(\bmod 3), \\
& T_{13 n+9} \equiv 0(\bmod 3) ;
\end{aligned}
$$

for the second sequence are valid:

$$
\begin{aligned}
T_{13 n} & \equiv 0(\bmod 9), \\
T_{13 n+1} & \equiv 0(\bmod 9), \\
T_{13 n+8} & \equiv 0(\bmod 3), \\
T_{13 n+10} & \equiv 0(\bmod 9) ;
\end{aligned}
$$

and for the third sequence are valid:

$$
\begin{aligned}
T_{13 n} & \equiv 0(\bmod 9), \\
T_{13 n+7} & \equiv 0(\bmod 3), \\
T_{13 n+9} & \equiv 0(\bmod 9), \\
T_{13 n+12} & \equiv 0(\bmod 9) .
\end{aligned}
$$

\section{Conclusion}

In a next research, we will check whether the 2-Fibonacci (see [1, 2, 5, 7]), 3-Fibonacci (see $[3,5])$ and 2-Tribonacci (see $[4,5])$ sequences have similar properties. 


\section{References}

[1] Atanassov, K., Atanassova, L., \& Sasselov, D. (1985). A new perspective to the generalization of the Fibonacci sequence. The Fibonacci Quarterly, 23 (1), 21-28.

[2] Atanassov, K. (1986). On a second new generalization of the Fibonacci sequence. The Fibonacci Quarterly, 24 (4), 362-365.

[3] Atanassov, K. (1989). On a generalization of the Fibonacci sequence in the case of three sequences. The Fibonacci Quarterly, 27 (1), 7-10.

[4] Atanassov, K., Hlebarova, J., \& Mihov, S. (1992). Recurrent formulas of the generalized Fibonacci and Tribonacci sequences. The Fibonacci Quarterly, 30 (1), 77-79.

[5] Atanassov, K., Atanassova, V., Shannon, A., \& Turner, J. (2002). New Visual Perspectives on Fibonacci Numbers. World Scientific, New Jersey.

[6] Feinberg, M. (1963). Fibonacci-Tribonacci. The Fibonacci Quarterly, 1 (3), 71-74.

[7] Lee, J.-Z.,\& Lee, J.-S. (1987). Some properties of the generalization of the Fibonacci sequence. The Fibonacci Quarterly, 25 (2), 111-117.

[8] Shannon, A. (1977). Tribonacci numbers and Pascal's pyramid. The Fibonacci Quarterly, 15 (3), 268-275.

[9] Spickerman, W. (1982). Binet's formula for the Tribonacci sequence. The Fibonacci Quarterly, 20 (2), 118-120. 